DOI: $10.2478 /$ v10324-012-0011-6<smiles>CC1=CC=C1</smiles>

VERSITA
Analele Universităţii de Vest,

Timişoara

Seria Matematică - Informatică

L, 2, (2012), 3- 14

\title{
On Uniform Exponential Dichotomy of Evolution Operators
}

Mihai Gabriel Babuţia, Traian Ceauşu, and Nicolae Marian Seimeanu

\begin{abstract}
The present paper presents three distinct concepts of uniform exponential dichotomy for evolution operators in Banach spaces. Characterizations and relationships between them and some illustrative counterexamples are given.
\end{abstract}

AMS Subject Classification (2000). 34D09

Keywords. exponential dichotomy; evolution operator.

\section{Preliminaries}

Let $X$ be a real or complex Banach space, and $\mathcal{B}(X)$ the algebra of bounded linear operators acting on $X$. We denote by $\|\cdot\|$ the norm on $X$ and on $\mathcal{B}(X)$, and let $\Delta$ be the set of all pairs of real nonnegative numbers $(t, s)$ satisfying $t \geq s$.

Definition 1.1. A map $U: \Delta \rightarrow \mathcal{B}(X)$ is called an evolution operator on $X$ if the following conditions hold:

e) $U(t, t)=I$, for all $t \geq 0$.

$\left.e_{2}\right) U(t, s) U\left(s, t_{0}\right)=U\left(t, t_{0}\right)$, for all $(t, s),\left(s, t_{0}\right) \in \Delta$.

The first author was supported by a grant of the Romanian National Authority for Scientific Research, CNCS UEFISCDI, project number PN-II-RU-TE-2011-3-0103. 
In addition,

(i) if there exist $M \geq 1$ and $\omega>0$ such that

$$
\|U(t, s) x\| \leq M e^{\omega(t-s)}\|x\| \quad \forall(t, s, x) \in \Delta \times X
$$

then we say that $U: \Delta \rightarrow \mathcal{B}(X)$ is an evolution operator with uniform exponential growth (and we write $U: \Delta \rightarrow \mathcal{B}(X)$ has (ueg));

(ii) if for all $(t, s) \in \Delta$ the operator $U(t, s)$ is bijective then we say that $U: \Delta \rightarrow \mathcal{B}(X)$ is reversible.

Definition 1.2. A map $P: \mathbb{R}_{+} \rightarrow \mathcal{B}(X)$ is called a family of projections on $X$ if

$$
P(t)^{2}=P(t), \quad \forall t \geq 0 .
$$

If $P: \mathbb{R}_{+} \rightarrow \mathcal{B}(X)$ is a family of projections on $X$ then $Q: \mathbb{R}_{+} \rightarrow \mathcal{B}(X)$ defined, for all $t \geq 0$ by

$$
Q(t)=I-P(t)
$$

is also a family of projections on $X$, called tht complementary projection of $P$.

Definition 1.3. We say that a family of projections $P: \mathbb{R}_{+} \rightarrow \mathcal{B}(X)$ is compatible with an evolution operator $U: \Delta \rightarrow \mathcal{B}(X)$ if the following condition holds

$$
U(t, s) P(s)=P(t) U(t, s), \quad \forall(t, s) \in \Delta .
$$

Remark 1.1. Let $U: \Delta \rightarrow \mathcal{B}(X)$ be an evolution operator and $P: \mathbb{R}_{+} \rightarrow$ $\mathcal{B}(X)$ a family of projections compatible with $U$. Then we have that

(i) for all $(t, s) \in \Delta, U(t, s) Q(s)=Q(t) U(t, s)$.

(ii) In addition, if $U: \Delta \rightarrow \mathcal{B}(X)$ is reversible, then for all $(t, s) \in \Delta$ we have that

$$
Q(s) U(t, s)^{-1}=U(t, s)^{-1} Q(t) .
$$

In what follows, for an evolution operator $U: \Delta \rightarrow \mathcal{B}(X)$ and a family of projections $P: \mathbb{R}_{+} \rightarrow \mathcal{B}(X)$, we will use the following notations:

$$
U_{P}(t, s)=U(t, s) P(s), \quad U_{Q}(t, s)=U(t, s) Q(s), \quad(t, s) \in \Delta .
$$

In this paper, three notions of uniform exponential dichotomy for evolution operators are introduced. The classical concept of exponential dichotomy for 
nonautonomous linear differential equations has been introduced by Perron [9] in the late 1920s. In this sequel, many authors developed the theory. For fundamental books we refer to Coppel [3], Massera and Schäffer [5], Daleckii and Krein [4], Barreira and Valls [1].

The first concept considered in this paper is the strong uniform exponential dichotomy. It is inspired by the [1] and [2]. The second, the uniform exponential dichotomy, is considered in [3], [4], [5], [6], [7] and [10].

We introduce a new concept of uniform exponential dichotomy which will be named weak uniform exponential dichotomy. It is implied by both of the preceding concepts and it is different from them.

The implications between these concepts are studied.

\section{Concepts of uniform exponential dichotomy}

A first concept of uniform exponential dichotomy is introduced by

Definition 2.1. Let $U: \Delta \rightarrow \mathcal{B}(X)$ be an evolution operator and $P: \mathbb{R}_{+} \rightarrow$ $\mathcal{B}(X)$ a family of projections. We say that $U$ is P-strongly uniformly exponentially dichotomic (and we write P-s.u.e.d) if there exist constants $N \geq 1, \nu>0$ such that for all $(t, s) \in \Delta$ the following hold:

$$
\begin{aligned}
& \text { (sued1) }\left\|U_{P}(t, s)\right\| \leq N e^{-\nu(t-s)} ; \\
& \text { (sued2) } N\left\|U_{Q}(t, s)\right\| \geq e^{\nu(t-s)}
\end{aligned}
$$

Remark 2.1. If $U: \Delta \rightarrow \mathcal{B}(X)$ is P-s.u.e.d then

$$
1 \leq\|P(t)\| \leq N, \quad 1 \leq\|Q(t)\| \leq 2 N \quad \forall t \geq 0
$$

The analogus of Definition 2.1 in the strong topology cannon give a proper dichotomy concept, the decomposition of the state space always being trivial as we will show below. Assume that there exist an evolution operator $U$ : $\Delta \rightarrow \mathcal{B}(X)$ and a family of projections $P: \mathbb{R}_{+} \rightarrow \mathcal{B}(X)$ such that there exist $N \geq 1$ and $\nu>0$ verifying

$$
N\left\|U_{Q}(t, s) x\right\| \geq e^{\nu(t-s)}\|x\|, \quad \forall(t, s, x) \in \Delta \times X .
$$

Let $t \geq 0$ and $s=t$ in the above estimation. It follows that

$$
N\|Q(t) x\| \geq\|x\|, \quad \forall x \in X
$$


which shows us that the projection $Q(t)$ is injective, hence

$$
\operatorname{Ran} P(t)=\operatorname{Ker} Q(t)=\{0\}, \quad \forall t \geq 0
$$

from where we get that $P(t)$ is the null operator for all $t \geq 0$ which means that the state space $X$ is not decomposed into two proper closed subspaces hence this concept characterizes a uniform exponential instability of the evolution operator $U: \Delta \rightarrow \mathcal{B}(X)$.

Definition 2.2. Let $U: \Delta \rightarrow \mathcal{B}(X)$ be an evolution operator and $P: \mathbb{R}_{+} \rightarrow$ $\mathcal{B}(X)$ a family of projections. We say that $U$ is P-uniformly exponentially dichotomic (and we write $P$-u.e.d) if there exist constants $N \geq 1$, $\nu>0$ such that for all $(t, s, x) \in \Delta \times X$ the following hold:

$$
\begin{aligned}
& \text { (ued1) }\left\|U_{P}(t, s) x\right\| \leq N e^{-\nu(t-s)}\|P(s) x\| ; \\
& \text { (ued2) } N\left\|U_{Q}(t, s) x\right\| \geq e^{\nu(t-s)}\|Q(s) x\| .
\end{aligned}
$$

Remark 2.2. Let $U: \Delta \rightarrow \mathcal{B}(X)$ be an evolution operator and $P: \mathbb{R}_{+} \rightarrow$ $\mathcal{B}(X)$ a family of projections. We have that $U$ is P-u.e.d if and only if there exist $N \geq 1$ and $\nu>0$ such that for all $(t, s, x) \in \Delta \times X$ the following condition holds:

$$
\left\|U_{P}(t, s) x\right\|+e^{\nu(t-s)}\|Q(s) x\| \leq N\left(e^{-\nu(t-s)}\|P(s) x\|+\left\|U_{Q}(t, s) x\right\|\right) .
$$

Proposition 2.1. If $U: \Delta \rightarrow \mathcal{B}(X)$ is a reversible evolution operator and $P: \mathbb{R}_{+} \rightarrow \mathcal{B}(X)$ is a family of projections compatible with $U$, then $U$ is $P$-u.e.d if and only if there exist $N \geq 1$ and $\nu>0$ such that the following conditions hold

(i) $\left\|U_{P}(t, s) x\right\| \leq N e^{-\nu(t-s)}\|P(s) x\|$

(ii) $\left\|U(t, s)^{-1} Q(t) x\right\| \leq N e^{-\nu(t-s)}\|Q(t) x\|$

for all $(t, s, x) \in \Delta \times X$.

Proof. Because (i) and (ued1) are the same, we only have to prove the equivalence $(u e d 2) \Leftrightarrow(i i)$. Assume that (ued2) holds. Then, for $(t, s, x) \in \Delta \times X$ we have that

$$
\begin{aligned}
\left\|U(t, s)^{-1} Q(t) x\right\| & =\left\|Q(s) U(t, s)^{-1} x\right\| \leq N e^{-\nu(t-s)}\left\|U_{Q}(t, s) U(t, s)^{-1} x\right\|= \\
& =N e^{-\nu(t-s)}\|Q(t) x\| .
\end{aligned}
$$

Conversely, for $(t, s, x) \in \Delta \times X$ we have, taking into account (ii), that

$$
\left\|U(t, s)^{-1} Q(t) U_{Q}(t, s) x\right\|=\|Q(s) x\| \leq N e^{-\nu(t-s)}\left\|U_{Q}(t, s) x\right\|
$$

(ued2) easily follows. 
Vol. L (2012) On Uniform Exponential Dichotomy...

Proposition 2.2. Let $U: \Delta \rightarrow \mathcal{B}(X)$ be an evolution operator and $P: \mathbb{R}_{+} \rightarrow$ $\mathcal{B}(X)$ a family of projections compatible with $U$. Then $U$ is P-u.e.d if and only if there exist $N \geq 1$ and $\nu>0$ such that for all $t \geq s \geq t_{0} \geq 0$ and for all $x \in X$ we have that

(i) $\left\|U_{P}\left(t, t_{0}\right) x\right\| \leq N e^{-\nu(t-s)}\left\|U_{P}\left(s, t_{0}\right) x\right\|$;

(ii) $N\left\|U_{Q}\left(t, t_{0}\right) x\right\| \geq e^{\nu(t-s)}\left\|U_{Q}\left(s, t_{0}\right) x\right\|$.

Proof. Necessity. Let $N \geq 1$ and $\nu>0$ given by Definition 2.2. For $t \geq s \geq$ $t_{0} \geq 0$ and $x \in X$ we have that

$$
\begin{aligned}
\left\|U_{P}\left(t, t_{0}\right) x\right\| & =\left\|U_{P}(t, s) U_{P}\left(s, t_{0}\right) x\right\| \leq \\
& \leq N e^{-\nu(t-s)}\left\|U_{P}\left(s, t_{0}\right) x\right\| ; \\
N\left\|U_{Q}\left(t, t_{0}\right) x\right\| & =N\left\|U_{Q}(t, s) U_{Q}\left(s, t_{0}\right) x\right\| \geq \\
& \geq e^{\nu(t-s)}\left\|U_{Q}\left(s, t_{0}\right) x\right\| .
\end{aligned}
$$

The sufficiency easily follows by putting $s=t_{0}$ in (i) and (ii).

Remark 2.3. It is well known (se for example [8]) that if and evolution operator $U: \Delta \rightarrow \mathcal{B}(X)$ has a uniform exponential growth and it is P-u.e.d then

$$
\sup _{t \geq 0}\|P(t)\|<+\infty .
$$

Definition 2.3. Let $U: \Delta \rightarrow \mathcal{B}(X)$ be an evolution operator and $P: \mathbb{R}_{+} \rightarrow$ $\mathcal{B}(X)$ a family of projections. We say that $U$ is $P$-weakly exponentially dichotomic (and we write P-w.u.e.d) if there exist constants $N \geq 1, \nu>0$ such that for all $(t, s) \in \Delta$ the following hold:

$$
\begin{aligned}
& \text { (wued1) }\left\|U_{P}(t, s)\right\| \leq N e^{-\nu(t-s)}\|P(s)\| \text {; } \\
& \text { (wued2) } N\left\|U_{Q}(t, s)\right\| \geq e^{\nu(t-s)}\|Q(s)\| .
\end{aligned}
$$

Proposition 2.3. Let $U: \Delta \rightarrow \mathcal{B}(X)$ be an evolution operator with (ueg) and $P: \mathbb{R}_{+} \rightarrow \mathcal{B}(X)$ a family of projections. If $U$ is $P$-w.u.e.d then

$$
\sup _{t \geq 0}\|P(t)\|<+\infty .
$$

Proof. Let $N, \nu$ given by the the P-w.u.e.d property of $U: \Delta \rightarrow \mathcal{B}(X)$, and $M, \omega$ given by the uniform exponential growth. Let $s \geq 0, t \geq s$. It follows that

$$
\begin{aligned}
& \left(\frac{1}{2 N} e^{\nu(t-s)}-N e^{-\nu(t-s)}\right)\|P(s)\| \leq \\
\leq & \frac{1}{N} e^{\nu(t-s)}\|Q(s)\|-N e^{-\nu(t-s)}\|P(s)\| \leq \\
\leq & \left\|U_{Q}(t, s)\right\|-\left\|-U_{P}(t, s)\right\| \leq\|U(t, s)\| \leq M e^{\omega(t-s)} .
\end{aligned}
$$


Let $\lambda_{0}>0$ be such that

$$
c_{0}:=\frac{1}{2 N} e^{\nu \lambda_{0}}-N e^{-\nu \lambda_{0}}>0 .
$$

Let in the above estimations $t=s+\lambda_{0}$. It follows that

$$
\|P(s)\| \leq \frac{M e^{\omega \lambda_{0}}}{c_{0}}, \quad \forall s \geq 0
$$

from where the conclusion easily follows.

Proposition 2.4. Let $U: \Delta \rightarrow \mathcal{B}(X)$ be an evolution operator and $P: \mathbb{R}_{+} \rightarrow$ $\mathcal{B}(X)$ a family of projections. If $U$ is P-s.u.e.d then $U$ is P-w.u.e.d.

Proof. Let $N \geq 1$ and $\nu>0$ be given by Definition 2.1. By Remark 2.1 it follows that

$$
\|Q(t)\| \leq 2 N, \quad \forall t \geq 0 .
$$

From (sued1) and (sued2), for $(t, s) \in \Delta$, we obtain that

$$
\begin{aligned}
\left\|U_{P}(t, s)\right\| & \leq N e^{-\nu(t-s)} \cdot 1 \leq N e^{-\nu(t-s)}\|P(s)\| \leq 2 N^{2} e^{-\nu(t-s)}\|P(s)\| ; \\
2 N^{2}\left\|U_{Q}(t, s)\right\| & \geq 2 N e^{\nu(t-s)} \geq e^{\nu(t-s)}\|Q(s)\|
\end{aligned}
$$

from where we deduce that $U: \Delta \rightarrow \mathcal{B}(X)$ is P-w.u.e.d with constants $2 N^{2} \geq 1$ and $\nu>0$.

Remark 2.4. Let $U: \Delta \rightarrow \mathcal{B}(X)$ be an evolution operator and $P: \mathbb{R}_{+} \rightarrow$ $\mathcal{B}(X)$ a family of projections. Then one has that if $U$ is P-u.e.d then $U$ is P-w.u.e.d

The converses of the above implications are not generally true, as we can see from the following examples.

Example 2.1. Consider the following evolution operators defined on $\mathbb{R}$ and $\mathbb{R}^{2}$ respectively, defined for all $(t, s) \in \Delta$ by

$$
\begin{aligned}
& U_{1}(t, s): \mathbb{R} \rightarrow \mathbb{R}, \quad U_{1}(t, s) x=e^{s-t} \cdot x \quad \forall x \in \mathbb{R} \\
& U_{2}(t, s): \mathbb{R}^{2} \rightarrow \mathbb{R}^{2}, \quad U_{2}(t, s) y=\left\{\begin{array}{ll}
y & t=s \\
\left(e^{t-s} y_{1}, 0\right) & t>s
\end{array} \quad \forall y=\left(y_{1}, y_{2}\right) \in \mathbb{R}^{2} .\right.
\end{aligned}
$$

On the Banach space $X=\mathbb{R} \oplus \mathbb{R}^{2}$ endowed with the norm

$$
\|x \oplus y\|=\left(|x|^{2}+\|y\|^{2}\right)^{1 / 2} \quad \forall x \oplus y \in X
$$


we define the family of projections $P: \mathbb{R}_{+} \rightarrow \mathcal{B}(X)$ by

$$
P(t) x \oplus y=x \oplus 0 \quad \forall(t, x \oplus y) \in \mathbb{R}_{+} \times X .
$$

For $(t, s) \in \Delta$ we consider the evolution operator

$$
U(t, s) x \oplus y=U_{1}(t, s) x \oplus U_{2}(t, s) y, \quad \forall x \oplus y \in X .
$$

We have that for all $(t, s, x \oplus y) \in \Delta \times X$,

$$
\begin{aligned}
& U_{P}(t, s) x \oplus y=U_{1}(t, s) x \oplus 0 \\
& U_{Q}(t, s) x \oplus y=0 \oplus U_{2}(t, s) y .
\end{aligned}
$$

It follows that for $(t, s) \in \Delta$ and $x \oplus y \in X$ we have that

$$
\left\|U_{P}(t, s) x \oplus y\right\|=\left|U_{1}(t, s) x\right|=e^{s-t}|x| \leq e^{s-t}\|x \oplus y\|
$$

from where we obtain that

$$
\left\|U_{P}(t, s)\right\| \leq e^{s-t}\|P(s)\|, \quad \forall(t, s) \in \Delta
$$

which shows us that (wued1) is fulfilled.

Moreover,

$$
\left\|U_{Q}(t, s) x \oplus y\right\|=\left\|U_{2}(t, s) y\right\| \leq e^{t-s}\|y\| \leq e^{t-s}\|x \oplus y\|
$$

for all $(t, s, x \oplus y) \in \Delta \times X$ from where we have

$$
\left\|U_{Q}(t, s)\right\| \leq e^{t-s} .
$$

Choosing $x \oplus y=0 \oplus(1,0) \in X$ with $\|x \oplus y\|=1$, we have that

$$
\left\|U_{Q}(t, s) x \oplus y\right\|=e^{t-s}\|x \oplus y\|
$$

from where we finally get that

$$
\left\|U_{Q}(t, s)\right\|=e^{t-s}=e^{t-s}\|Q(s)\|, \quad \forall(t, s) \in \Delta
$$

which shows us that condition (wued2) is fulfilled hence $U: \Delta \rightarrow \mathcal{B}(X)$ is P-w.u.e.d.

Assume by a contradiction that $U: \Delta \rightarrow \mathcal{B}(X)$ is P-u.e.d. Then in particular there exist $N \geq 1$ and $\nu>0$ such that

$$
N\left\|U_{Q}(t, s) x \oplus y\right\| \geq e^{\nu(t-s)}\|Q(s) x \oplus y\|, \quad \forall(t, s, x \oplus y) \in \Delta \times X .
$$

Let $t>s$ and $x \oplus y=0 \oplus(0,1)$. Then $\|Q(s) x \oplus y\|=1$ and

$$
e^{\nu(t-s)} \leq\left\|U_{Q}(t, s) x \oplus y\right\|=\|U(t, s) 0 \oplus(0,1)\|=0
$$

which is a contradiction. 
We saw that in the general case, the concepts of P-u.e.d and P-w.u.e.d. do not coincide. We may ask ourselves wether in the case of reversible operators the converse implication from Remark 2.4 holds.

The answer is negative, and it is given by the following example.

Example 2.2. On $X=\mathbb{R}^{3}$ endowed with the max-norm

$$
\left\|\left(x_{1}, x_{2}, x_{3}\right)\right\|=\max \left\{\left|x_{1}\right|,\left|x_{2}\right|,\left|x_{3}\right|\right\}, \quad \forall\left(x_{1}, x_{2}, x_{3}\right) \in \mathbb{R}^{3}
$$

consider, for $(t, s) \in \Delta$ the operator $U(t, s): \mathbb{R}^{3} \rightarrow \mathbb{R}^{3}$ defined by

$$
U(t, s)\left(x_{1}, x_{2}, x_{3}=\left(e^{s-t} x_{1}, e^{t-s} x_{2}, x_{3}\right) \quad \forall\left(x_{1}, x_{2}, x_{3}\right) \in \mathbb{R}^{3} .\right.
$$

Define $P: \mathbb{R}_{+} \rightarrow \mathcal{B}(X)$ by

$$
P(t)\left(x_{1}, x_{2}, x_{3}\right)=\left(x_{1}, 0,0\right), \quad \forall\left(x_{1}, x_{2}, x_{3}\right) \in \mathbb{R}^{3}, \quad \forall t \geq 0 .
$$

We have that $U: \Delta \rightarrow \mathcal{B}(X)$ is a reversible evolution operator and $P: \mathbb{R}_{+} \rightarrow$ $\mathcal{B}(X)$ is compatible with $U$ having

$$
U(t, s)^{-1}\left(x_{1}, x_{2}, x_{3}\right)=\left(e^{t-s} x_{1}, e^{s-t} x_{2}, x_{3}\right) \quad \forall\left(x_{1}, x_{2}, x_{3}\right) \in \mathbb{R}^{3} .
$$

We will prove that $U$ is w.u.e.d with $N=\nu=1$.

Let $(t, s) \in \Delta$. From

$$
\left\|U_{P}(t, s)\left(x_{1}, x_{2}, x_{3}\right)\right\|=e^{s-t} P(s)\left(x_{1}, x_{2}, x_{3}\right), \quad \forall\left(x_{1}, x_{2}, x_{3}\right) \in \mathbb{R}^{3}
$$

we obtain that

$$
\left\|U_{P}(t, s)\right\|=e^{s-t}\|P(s)\|
$$

which shows us that condition (wued1) is satisfied.

Moreover, from

$$
\left\|U_{Q}(t, s)\left(x_{1}, x_{2}, x_{3}\right)\right\|=\left(0, e^{t-s} x_{2}, x_{3}\right)
$$

it follows that

$$
\left\|U_{Q}(t, s)\left(x_{1}, x_{2}, x_{3}\right)\right\|=\max \left\{e^{t-s}\left|x_{2}\right|,\left|x_{3}\right|\right\} \leq e^{t-s}\|Q(s) x\|
$$

from where we get that

$$
\left\|U_{Q}(t, s)\right\| \leq e^{t-s}\|Q(s)\|
$$

On the other hand, consider the vector $(0,1,0) \in \mathbb{R}^{3}$. It follows that

$$
\left\|U_{Q}(t, s)(0,1,0)\right\|=e^{t-s}=e^{t-s}\|Q(s) x\|
$$


from where we finally get that

$$
\left\|U_{Q}(t, s)\right\|=e^{t-s}\|Q(s)\|
$$

which shows us that condition (wued2) is fulfilled.

Assume now that $U: \Delta \rightarrow \mathcal{B}(X)$ is P-u.e.d. From (ued2) it follows that there exist $N \geq 1$ and $\nu>0$ such that

$$
N\left\|U_{Q}(t, s) x\right\| \geq e^{\nu(t-s)}\|Q(s) x\|
$$

for all $(t, s, x) \in \Delta \times X$. Having in mind that

$$
Q(s)(0,0,1)=(0,0,1), \quad U_{Q}(t, s)(0,0,1)=(0,0,1)
$$

taking in account (2.3) we get that

$$
N \geq e^{\nu(t-s)}, \quad \forall(t, s) \in \Delta
$$

which led us to a contradiction.

Remark 2.5. In the above example, having in mind that

$$
\|P(t)\|=\|Q(t)\|=1, \quad \forall t \geq 0
$$

from the relations (2.1) and (2.2) we get that

$$
\left\|U_{P}(t, s)\right\|=e^{s-t}, \quad\left\|U_{Q}(t, s)\right\|=e^{t-s}, \quad \forall(t, s) \in \Delta
$$

hence the evolution operator corresponding to this example is P-s.u.e.d but fails to be P-u.e.d.

Seeing that P-s.u.e.d does not generally imply P-u.e.d, we may ask ourselves wether the P-u.e.d property implies P-s.u.e.d. The answer is negative, and we point out this fact through the following example.

Example 2.3. Let $X=\mathbb{R}^{2}$ endowed with the max-norm

$$
\|x\|=\max \left\{\left|x_{1}\right|,\left|x_{2}\right|\right\}, \quad x=\left(x_{1}, x_{2}\right) \in \mathbb{R}^{2} .
$$

For every $t \geq 0$, we define

$$
P(t): \mathbb{R}^{2} \rightarrow \mathbb{R}^{2}, P(t)\left(x_{1}, x_{2}\right)=\left(x_{1}+t x_{2}, 0\right), \quad \forall\left(x_{1}, x_{2}\right) \in \mathbb{R}^{2} .
$$

We have that $P: \mathbb{R}_{+} \rightarrow \mathcal{B}(X)$ is a family of projections and

$$
\|P(t)\|=1+t, \quad \forall t \geq 0 .
$$


For $(t, s) \in \Delta$ we define $U(t, s): \mathbb{R}^{2} \rightarrow \mathbb{R}^{2}$ by

$$
U(t, s) x=e^{s-t} P(s) x+e^{t-s} Q(t) x, \quad \forall x \in \mathbb{R}^{2} .
$$

Having in mind that $P(t) P(s)=P(s), Q(t) Q(s)=Q(t)$ and $Q(t) P(s)=0$ for all $t, s \in \mathbb{R}_{+}$, it easily follows that $U: \Delta \rightarrow \mathcal{B}(X)$ is an evolution operator. We observe that for all $\left(t, s, x_{1}, x_{2}\right) \in \Delta \times \mathbb{R}^{2}$ we have

$$
\left\|Q(s)\left(x_{1}, x_{2}\right)\right\|=\max \{s, 1\}\left|x_{2}\right| \leq \max \{t, 1\}\left|x_{2}\right|=\left\|Q(t)\left(x_{1}, x_{2}\right)\right\| .
$$

Moreover, we have that for all $(t, s, x) \in \Delta \times X$,

$$
\left\|U_{P}(t, s) x\right\|=e^{-(t-s)}\|P(s) x\|,\left\|U_{Q}(t, s) x\right\|=e^{t-s}\|Q(t) x\| \geq e^{t-s}\|Q(s) x\|
$$

which shows us that $U$ is $\mathrm{P}$-u.e.d.

Assuming by a contradiction that $U$ is P-s.u.e.d, by Remark 2.1 we would have that there exists $K>0$ such that

$$
\|P(t)\| \leq K, \quad \forall t \geq 0
$$

which obviously does not hold.

Remark 2.6. Considering the evolution operator $U: \Delta \rightarrow \mathcal{B}(X)$ and the family of projections from Example 2.3, the relations from (2.4) imply that

$$
\left\|U_{P}(t, s)\right\|=e^{s-t}\|P(s)\|, \quad\left\|U_{Q}(t, s)\right\| \geq e^{t-s}\|Q(s)\| \quad \forall(t, s) \in \Delta
$$

hence $U$ is $\mathrm{P}$-w.u.e.d. The same contradiction is obtained by assuming that $U$ is P-s.u.e.d.

Remark 2.7. Having in mind all of the above, we can point out the diagram concerning the connections between the presented concepts:

$$
\begin{array}{ccc} 
& P \text { - s.u.e.d } \\
\not \notin & & \Downarrow \\
P-\text { u.e.d } & \Longrightarrow & P-\text { w.u.e.d }
\end{array}
$$

\section{Acknowledgement}

The authors want to thank Prof. Dr. Mihail Megan, the head of Research Seminar of Mathematical Analysis and Applications, who proposed to solve these problems. 


\section{References}

[1] L. Barreira and C. Valls, Stability of Nonautonomous Differential Equations, Lecture Notes in Mathematics 1926, 2008

[2] C. Chicone and Y Latushkin, Evolution Semigroups in Dynamical Systems and Differential Equations, Math. Surveys and Monographs, vol. 70, Amer. Math. Soc., 1999

[3] W. A. Coppel, Stability and asymptotic behaviour of differential equations, D.C. Heath, Boston, 1965

[4] J. L. Daleckii and M. G. Krein, Stability of differential equations in Banach space, Amer. Math. Soc., Providence, R. I., 1974

[5] J. L. Massera and J. J. Schäffer, Linear differential equations and function spaces, Academic Press, New York and London, 1966

[6] M. Megan, On (h,k)-dichotomy of evolution operators in Banach spaces, Dynamic Systems and Applications, 5, (1996), 189-196

[7] M. Megan, B. Sasu, and A. L. Sasu, On nonuniform exponential dichotomy of evolution operators in Banach spaces, Integral Equations Operator Theory, 44, (2002), $71-78$

[8] N. Van Minh, F. Räbiger, and R. Schnaubelt, Exponential stability, exponential expansiveness and exponential dichotomy of evolution equations on the half-line, Integr. Equ. Oper. Theory, 32, (1998), 332-353

[9] O. Perron, Die stabilitätsfrage bei Differentialgleischungen, Math. Z., 32, (1930), 703-728

[10] P. Preda and M. Megan, Exponential dichotomy of evolutionary processes in Banach spaces, Czechoslovak Mathematical Journal, 35 (2), (1985), 312-323

Mihai Gabriel Babutia

Department of Mathematics

West University of Timişoara

V. Pârvan Blvd. No. 4

300223 Timişoara

Romania

E-mail: mbabutia@math.uvt.ro

Traian Ceauşu

Department of Mathematics

West University of Timişoara

V. Pârvan Blvd. No. 4

300223 Timişoara

Romania

E-mail: ceausu@math.uvt.ro 
Nicolae Marian Seimeanu

Department of Mathematics

West University of Timişoara

V. Pârvan Blvd. No. 4

300223 Timişoara

Romania

E-mail: nicusei@yahoo.com

Received: 28.07.2012

Accepted: 15.11.2012 